LETTER

The local vicinity of spin sums for certain massdimension-one spinors

To cite this article: R. J. Bueno Rogerio and J. M. Hoff da Silva 2017 EPL 11810003

View the article online for updates and enhancements.
Related content

Constraining Elko dark matter at the LHC with monophoton events

Alexandre Alves, M. Dias, F. de Campos

et al.

Evading Weinberg's no-go theorem to construct mass dimension one fermions: Constructing darkness Dharam Vir Ahluwalia

The connection between Dirac dynamic and parity symmetry

C. H. Coronado Villalobos and R. J. Bueno Rogerio

\section{Recent citations}

- The restricted Inomata-McKinley spinor-
plane, homotopic deformations and the
$\frac{\text { Lounesto classification }}{\text { D. Beghetto et al }}$
- The Heisenberg spinor field classification
$\frac{\text { and its interplay with the Lounesto's }}{\text { classification }}$
Marcos R. A. Arcodía et al
- Further investigation of mass dimension
one fermionic duals
J.M. Hoff da Silva and R.T. Cavalcanti




\title{
The local vicinity of spin sums for certain mass-dimension-one spinors
}

\author{
R. J. Bueno Rogerio and J. M. Hoff Da Silva \\ Departamento de Física e Química, Universidade Estadual Paulista - Guaratinguetá, SP, Brazil
}

received 7 April 2017; accepted in final form 12 May 2017

published online 30 May 2017

PACS $03.70 .+k-$ Theory of quantized fields

PACS 11.10.-z - Field theory

\begin{abstract}
The usual formulations of Elko is well known to lead to a subtle Lorentz symmetry breaking encoded in the spin sums. Recently it was proposed a redefinition in the dual structure, along with a given mathematical device, which eliminates the Lorentz breaking term in the spin sums. In this work we delve into the analysis of this mathematical device providing a solid framework to the used method.
\end{abstract}

Copyright (C) EPLA, 2017

Introduction. - Quite recently, a spin one-half fermionic field endowed with canonical mass dimension one which is local was proposed in [1], in contrary of what happens with the previous formulations $[2,3]$. The whole construction rests upon a remaining degree of freedom in the dual definition. Such freedom circumvents the rigidity of Weinberg's no-go theorem concerning spin one-half fermions [4].

The new dual possibility can be obtained from a twostep program: firstly, working out a non-vanishing real Lorentz invariant associated norm, secondly, evaluating the spin sums and imposing Lorentz covariance in the final result. In order to accomplish the second step, two more requirements are necessary: a redefinition on the dual structure, keeping all the previous physical contents, and what is being called by spin sums $\tau$-deformation $[1,5]$. After all, these procedures lead to a local fermionic field.

The aim of this paper is to formalize the last step, by means of the concept of the vicinity of singular matrix. We also delve into the failure of usual methods such as the Moore-Penrose pseudoinverse [6,7] (or generalized inverse) and Tikhonov's regularization [8]. This work is organized as follows: in the next section we review the basic points in the derivation of the new dual. Going forward, in the third section we explicit the problem concerning the Lorentz covariance of the spin sums and formalize the procedure performed in ref. [1], putting the new spin sums in a robust context. In the final section we conclude.

The dual. - There is a judicious program leading to an adequate dual associated to a given spinor field [9]. For bookkeeping purposes we shall depict the main aspects of this formulation. Let $\psi$ be a given spinor field carrying a $(1 / 2,0) \oplus(0,1 / 2)$ representation. Suppose that its dual is given by $\tilde{\psi}$. In order to reach the appropriate dual, consider the following general form:

$$
\tilde{\psi}_{h}\left(p^{\mu}\right)=\left[\Delta \psi_{h}\left(p^{\mu}\right)\right]^{\dagger} \eta,
$$

where $h$ stands for the helicity, such construction must yield a real and non-null Lorentz invariant norm. The $\Delta$ operator must obey a set of requirements. Denoting the spinor space by $S$, then the $\Delta$ operator is such that

$$
\begin{aligned}
\Delta: S & \rightarrow S, \\
\psi_{h} & \mapsto \psi_{h^{\prime}} .
\end{aligned}
$$

Besides, $\Delta^{2}=\mathbb{I}$ and $\Delta^{-1}$ have to exist, ensuring an invertible mapping [1]. Lorentz invariance imply $\eta \sim \gamma_{0}$, and from eq. (2) we may have two possibilities: $h=h^{\prime}$, for which $\Delta=\mathbb{I}$ and it stands for the Dirac usual case, or $h \neq h^{\prime}$ leading to a more involved operator, which may be represented by [1] $\Delta=m^{-1} G(\phi) \gamma_{\mu} p^{\mu}$, being $m$ the rest mass and $p^{\mu}$ the four momenta associated to the particle to be described by the quantum field. Moreover, the $G(\phi)$ matrix reads

$$
\mathcal{G}(\phi)=\left(\begin{array}{cccc}
0 & 0 & 0 & -i e^{-i \phi} \\
0 & 0 & i e^{i \phi} & 0 \\
0 & -i e^{-i \phi} & 0 & 0 \\
i e^{i \phi} & 0 & 0 & 0
\end{array}\right)
$$

where $\phi$ is the polar angle in the momentum parameterization $p^{\mu}=(E, p \sin \theta \cos \phi, p \sin \theta \sin \phi, p \cos \theta)$. Finally, 
the formal structure of the spinor must be evinced. Hence, keeping in mind the desired canonical mass dimension, and the neutrality of the resulting field, it is possible to see that the spinor field must be an eigenspinor of the charge conjugation operator $[1,2] C \psi^{S / A}\left(p^{\mu}\right)= \pm \psi^{S / A}\left(p^{\mu}\right)$, where $\psi^{S}\left(\psi^{A}\right)$ means (anti)self-conjugated spinor.

The aforementioned reasoning leads, after a bit of calculation, to the following spin sums:

$$
\sum_{h} \psi_{h}^{S / A}\left(p^{\mu}\right) \tilde{\psi}_{h}^{S / A}\left(p^{\mu}\right)= \pm m[\mathbb{I} \pm G(\phi)],
$$

revealing a subtle breaking in the Lorentz covariance. Here it is where the usual Elko construction stops. In the next section we shall pursue the dual redefinition and accomplish the formal $\tau$-deformation.

The spin sums inverse. - An attempt to solve the Elko breaking Lorentz covariance that emerges in the spin sums is given by a redefinition in the dual structure, as can be seen in ref. [1]. Such redefinition reads

$$
\begin{aligned}
\tilde{\psi}_{h}^{S}\left(p^{\mu}\right) & \rightarrow \tilde{\psi}_{h}^{S}\left(p^{\mu}\right) \mathcal{A}, \\
\tilde{\psi}_{h}^{A}\left(p^{\mu}\right) & \rightarrow \tilde{\psi}_{h}^{A}\left(p^{\mu}\right) \mathcal{B},
\end{aligned}
$$

where the operators $\mathcal{A}$ and $\mathcal{B}$ demand to have some important properties: the spinors $\psi_{h}^{S}\left(p^{\mu}\right)$ and $\psi_{h}^{A}\left(p^{\mu}\right)$ must to be eigenspinors of $\mathcal{A}$ and $\mathcal{B}$, respectively, with eigenvalues given by the unity

$$
\mathcal{A} \psi_{h}^{S}\left(p^{\mu}\right)=\psi_{h}^{S}\left(p^{\mu}\right), \quad \mathcal{B} \psi_{h}^{A}\left(p^{\mu}\right)=\psi_{h}^{A}\left(p^{\mu}\right) .
$$

Besides, such operators must fulfill

$$
\tilde{\psi}_{h}^{S}\left(p^{\mu}\right) \mathcal{A} \psi_{h}^{A}\left(p^{\mu}\right)=0, \quad \tilde{\psi}_{h}^{A}\left(p^{\mu}\right) \mathcal{B} \psi_{h}^{S}\left(p^{\mu}\right)=0 .
$$

The set of equations (7) and (8) ensures the accuracy of the orthonormality relations, as remarked in $[2,3]$, to remain unchanged. With the new dual structure in mind one can evaluate the spin sums, which now will carry the operators $\mathcal{A}$ and $\mathcal{B}$. A direct calculation leads to

$$
\begin{aligned}
\sum_{h} \psi_{h}^{S}\left(p^{\mu}\right) \tilde{\psi}_{h}^{S}\left(p^{\mu}\right) & =m[\mathbb{I}+G(\phi)] \mathcal{A} \\
\sum_{h} \psi_{h}^{A}\left(p^{\mu}\right) \tilde{\psi}_{h}^{A}\left(p^{\mu}\right) & =-m[\mathbb{I}-G(\phi)] \mathcal{B} .
\end{aligned}
$$

Now, in order to acquire Lorentz covariant spin sums, it could be imposed that $\mathcal{A}$ and $\mathcal{B}$ are simply the inverse of $[\mathbb{I}+G(\phi)]$ and $[\mathbb{I}-G(\phi)]$, respectively [1]. However, $\operatorname{det}[\mathbb{I} \pm G(\phi)]=0$ and this naive approach does not work. An interesting general inverse was introduced in the literature by the outstanding work of Moore [6] and further developed by Penrose [7]. On general grounds these works give a complete algorithm to find out the so-called pseudoinverse, hereafter denoted by $M^{+}$, of a singular matrix $M$.

As already noted in ref. [5], unfortunately this procedure appears to be innocuous. In fact, by applying the pseudoinverse protocol, the $[\mathbb{I}+\mathcal{G}(\phi)]^{+}$is given by $[7]$

$$
[\mathbb{I}+\mathcal{G}(\phi)]^{+}=[\mathbb{I}+\mathcal{G}(\phi)]^{\dagger}\left([\mathbb{I}+\mathcal{G}(\phi)][\mathbb{I}+\mathcal{G}(\phi)]^{\dagger}\right)^{-1}
$$

or, in a similar fashion,

$$
[\mathbb{I}+\mathcal{G}(\phi)]^{+}=\left([\mathbb{I}+\mathcal{G}(\phi)]^{\dagger}[\mathbb{I}+\mathcal{G}(\phi)]\right)^{-1}[\mathbb{I}+\mathcal{G}(\phi)]^{\dagger} .
$$

Nevertheless, both resulting amounts are singular, invalidating this approach. It is necessary, then, to look for an alternative method.

The method known as Tikhonov's regularization [8] provides another way to calculate the inverse of a singular matrix, given the failure of the previous formulation. Adapting this method to the problem at hand, one is lead to

$[\mathbb{I}+\mathcal{G}(\phi)]^{+}=\lim _{\tau \rightarrow 0}[\mathbb{I}+\mathcal{G}(\phi)]^{\dagger}\left([\mathbb{I}+\mathcal{G}(\phi)][\mathbb{I}+\mathcal{G}(\phi)]^{\dagger}+\mu \mathbb{I}\right)^{-1}$

where $\mu \in \mathbb{C}$ is a deformation-like parameter. A fairly trivial calculation allows one to recast the above equation as

$$
[\mathbb{I}+\mathcal{G}(\phi)]^{+}=\lim _{\mu \rightarrow 0}\left(\begin{array}{cccc}
\frac{1}{4+\mu} & 0 & 0 & \frac{-i e^{-i \phi}}{4+\mu} \\
0 & \frac{1}{4+\mu} & \frac{i e^{i \phi}}{4+\mu} & 0 \\
0 & \frac{-i e^{-i \phi}}{4+\mu} & \frac{1}{4+\mu} & 0 \\
\frac{i e^{i \phi}}{4+\mu} & 0 & 0 & \frac{1}{4+\mu}
\end{array}\right) .
$$

In order to verify if (13) matches the pseudoinverse of $[\mathbb{I}+G(\phi)]$, we multiply $[\mathbb{I}+\mathcal{G}(\phi)]^{+}$by $[\mathbb{I}+\mathcal{G}(\phi)]$ and take the limit $\mu \rightarrow 0$. As can be readily verified, the result is

$$
\begin{aligned}
{[\mathbb{I}+\mathcal{G}(\phi)][\mathbb{I}+\mathcal{G}(\phi)]^{+} } & =\frac{1}{2}\left(\begin{array}{cccc}
1 & 0 & 0 & -i e^{-i \phi} \\
0 & 1 & i e^{i \phi} & 0 \\
0 & -i e^{-i \phi} & 1 & 0 \\
i e^{i \phi} & 0 & 0 & 1
\end{array}\right) \\
& =\frac{1}{2}[\mathbb{I}+\mathcal{G}(\phi)]
\end{aligned}
$$

leading, again, to an inefficient method.

Aiming to find a matrix that can be really classified as an inverse to the Lorentz breaking part of the spin sums, we used a formulation called " $\tau$-deformation" [1], writing

$$
\sum_{h} \psi_{h}^{S}\left(p^{\mu}\right) \tilde{\psi}_{h}^{S}\left(p^{\mu}\right)=\left.m[\mathbb{I}+\tau G(\phi)] \mathcal{A}\right|_{\tau \rightarrow 1}
$$

and

$$
\sum_{h} \psi_{h}^{A}\left(p^{\mu}\right) \tilde{\psi}_{h}^{A}\left(p^{\mu}\right)=-\left.m[\mathbb{I}-\tau G(\phi)] \mathcal{B}\right|_{\tau \rightarrow 1} .
$$

Notice that in order for the $\tau$-deformation to make sense it must have a well-defined $\tau \rightarrow 1$ limit. Therefore, this 
limit is the unique necessary constraint used. The investigation of the vicinity of $[\mathbb{I}+\mathcal{G}(\phi)][10]$ corroborates this approach, giving full sense to the adopted inverse.

Guided by the same protocol as done in [10], we start from the fact that both matrices $\mathbb{I}$ and $\mathcal{G}(\phi)$ are nonsingular ${ }^{1}$. Once it is true, there exists constant numbers $r_{1}$ and $r_{2}$, which, in turn, depend on $\mathbb{I}$ and $\mathcal{G}(\phi)$, under the condition $0<r_{1} \leq r_{2}$, such that $\mathbb{I}+\tau \mathcal{G}(\phi)$ is invertible for all $\tau \in \mathbb{C}$ with $0<|\tau|<r_{1}$ and for all $\tau \in \mathbb{C}$ with $|\tau|>r_{2}$. Hence it is possible to see that

$$
\mathbb{I}+\tau G(\phi)=\left[\mathbb{I} \tau+G^{-1}(\phi)\right] G(\phi),
$$

in such a way that $[\mathbb{I}+\tau G(\phi)]$ is invertible only if $[\mathbb{I} \tau+$ $\left.G^{-1}(\phi)\right]$ is non-singular. Now take $Z \equiv-G^{-1}(\phi)$ and $\left\{\lambda_{1}, \ldots \lambda_{k}\right\} \in \mathbb{C}$ to be the $k$ not necessarily distinct roots of the polynomial $p_{z}$, i.e., $p_{z}=\operatorname{det}[Z-\lambda \mathbb{I}]$. Notice from eq. (17) that

$$
\mathbb{I}+\tau G(\phi)=-[Z-\mathbb{I} \tau] G(\phi) .
$$

If all roots are null, one should take $r_{1}=r_{2}$ as any positive number. Defining, then, $r_{1} \equiv \min \left\{\left|\lambda_{k}\right|, \lambda_{k} \neq 0\right\}$ and $r_{2} \equiv$ $\max \left\{\left|\lambda_{k}\right|\right\}$, we have two distinct ranges of values for $\tau$ (exactly the ranges without roots of $p_{z}$ ) for which $\tau$ enables $\left[\mathbb{I} \tau+\mathcal{G}^{-1}(\phi)\right]$ to be non-singular, ensuring $[\mathbb{I}+\tau G(\phi)]$ to be invertible. These ranges are $\left\{\tau \in \mathbb{R}, 0<|\tau|<r_{1}\right\}$ and $\left\{\tau \in \mathbb{R},|\tau|>r_{2}\right\}$. As can be seen,

$$
p_{z}=\lambda^{4}-2 \lambda^{2}+1
$$

have roots given by \pm 1 , both with multiplicity two. Therefore, one can see that the unique constraint is given by $|\tau|>1$ or $0<|\tau|<1$. Hence, the limit used in ref. [1] is indeed valid and the operators $\mathcal{A}$ and $\mathcal{B}$ may well be chosen in order to give the inverse of (15) and (16), respectively.

Final remarks. - We have studied some well-defined methods, aiming to find the inverse of a singular matrix emerging in the spin sums related to the Elko spinors usual formulation. After trying the standard methods encoded in the Moore-Penrose pseudoinverse and Tikhonov's regularization, we turned to the study of formalizations concerning the neighborhood of singular matrices. Within this context, we found that the $\tau$-deformation provides an acceptable inverse (also) in the limit $\tau \rightarrow 1$. It turns out that this same limit is necessary in the $\tau$-deformation used in ref. [1]. Therefore, the study of the vicinity of the Elko spin sums places the method used in [1] on a well-posed mathematical level.

The authors express their gratitude to Professor RoldÃO DA Rocha for the privilege of his revision, comments and appreciation of this work. JMHDS would like to thanks CNPq (445385/2014-6; 304629/2015-4) for partial support and RJBR thanks CAPES for the financial support.

\section{REFERENCES}

[1] Ahluwalia D. V., The Theory of Local Mass Dimension One Fermions of Spin One Half, to be published in Adv. Appl. Clifford Algebras (2017).

[2] Ahluwalia-Khalilova D. V. and Grumiller D., JCAP, 07 (2005) 012.

[3] Ahluwalia-Khalilova D. V. and Grumiller D., Phys. Rev. D, 72 (2005) 067701.

[4] Weinberg S., Phys. Rev., 133 (1964) B1318.

[5] Lee C.-Y., Phys. Lett. B., 760 (2016) 164.

[6] Moore E. H., Bull. Am. Math. Soc., 26 (1920) 394.

[7] Penrose R., Proc. Cambridge Philos. Soc., 51 (1955) 406.

[8] Tikhonov A. N., Sov. Math. Dokl., 4 (1963) 1035; Dokl. Akad. Nauk USSR, 151 (1963) 501.

[9] Ahluwalia D. V., Lee C.-Y. and Schritt D., Phys. Lett. B, 687 (2010) 248.

[10] Barata J. C. A. and Hussein M. S., Braz. J. Phys., 42 (2012) 146.

\footnotetext{
${ }^{1}$ We remark, parenthetically, that the same protocol and conclusions can be mutatis mutandis applied to the $[\mathbb{I}-G(\phi)]$ case.
} 